A. Pramesh Rao, G. Swarup and Gopal-Krishna, eds.

\title{
Concepts and Technical Studies of the Square Kilometre Array
}

\author{
Arnold van Ardenne \\ Netherlands Foundation for Research in Astronomy (ASTRON), \\ P.O.Box 2, 7990AA, Dwingeloo, The Netherlands
}

\begin{abstract}
We present the concepts and their unique features presently being studied for the Square Kilometer Array expected to be operational between 2010 and 2015. A comparison is made between telescopes in operation to day and SKA ambitious set of requirements is presented. The framework for coordinating the studies of various countries aiming to conclude on a concept choice in 5 years is introduced. As a desirable side effect, efforts relevant for SKA, will result in new albeit smaller telescopes coming into operation at earlier stages.
\end{abstract}

\section{Introduction}

Studies are being done to the radio telescope now called the Square Kilometre Array $[1,2]$ that will lead the onset of the new century. This instrument will image celestial radio sources based on the principle of earth rotation synthesis. In time it will concentrate on the science questions made possible by its orders of magnitude increase in sensitivity together with a large field of view, three decades frequency coverage and large resolution range among others.

Generally felt desirable to be fully operational in the second decade, the period of up to say, ten years is used for setting up the international framework, organise the community of technologists and addressing the main $\mathrm{R} \& \mathrm{D}$ issues at the appropriate level and identify the funding schemes. The effort is complementary when seen in the larger framework of astrophysics and instrumental developments in other i.e. (sub) $\mathrm{mm}$ and optical/IR wavelengths regimes that will precede SKA.

At occasions like this conference, aspects of immanent importance to the technical realisation of SKA are addressed in a wider community of users. Broadly speaking, these aspects cover new antenna concepts and new and enabling technologies in fields ranging from broadband antennas, low noise (integrated) frontends, photonics, signal processing and packaging in electronic Industry to calibration, interference mitigation strategies and image processing [3 and references].

Various countries agreed that an appropriate level of $\mathrm{R} \& \mathrm{D}$ would make a balanced choice between concepts around 2005 possible. A recently erected body will steer, monitor and facilitate progress toward an agreed first level set of requirements, and a concept design and architecture. 
Here, we will emphasise the different approaches and antenna technologies for SKA in view of some important system requirements. Many of the concepts are new in major aspects and have never been applied in radio astronomy. $\mathrm{Al}$ though highly ambitious in scope and desired performance, SKA at the same time relies on technologies and trends because of needs in other areas e.g. in wireless telecommunications and in general ICT applications hence building confidence in its feasibility.

\section{Limitations and Requirements}

Imaging radio astronomy relies heavily on incremental improvements of modular subsystems of long living observing platforms often based on $>25 \mathrm{~m}$ paraboloidal reflectors at $\mathrm{cm}$ and longer wavelengths. In parallel, Very Long Baseline Interferometry evolved over several decades with baselines now reaching out in space through an earth orbiting radiotelescope. These hard- and software developments are making possible images of radio sources up to a 100.000:1 dynamic range (defined as the ratio of strongest to weakest source in the image) on a routine basis together with calibrated large field surveys covering a few $\mathrm{MHz}$ of instantaneous spectrum. The long baselines in VLBI, resulted in sub-milliarcsecond resolution at $30 \mathrm{GHz}$ i.e. orders of magnitude better than telescopes in other wavelength regimes. Combined with improved stability and calibration techniques, radio astronomical systems thus remain state-of- the-art instruments for years to come.

Limitations arise because existing telescopes typically have collecting areas below $10.000 \mathrm{~m}^{2}$ while observing with a single beam in a relatively small number of protected but narrow frequency bands.

The (combined) requirements of SKA are difficult to achieve and the use of new technologies and design methodologies is required. To start of with, different novel antenna (system) concepts or combinations of these each having their own merits, are actively being studied in nationally supported programs in different countries.

Irrespective of these various concepts, some system level issues pertain to astronomical requirements that are difficult to translate into a set of clear engineering specifications. For example, the required sensitivity in large dynamic range images in view of RF-interference and over a decade or more frequency span. In particular at the lower frequencies, the issue of (ionosperic and other) calibration and the subsequent system design consequences [5] are relevant.

With regard to RFI-mitigation, activities of the astronomical community are directed toward sharing frequency space with other active and passive users based on cultural and other considerations. In parallel, $R \& D$ efforts exploit new techniques to reduce adverse effects for astronomy to be addressed with SKA. In today wireless society, results are relevant to other communities outside astronomy.

\section{The Concepts as they Evolve}

SKA was conceived as a single concept instrument of at least a decade bandwidth. Its collecting area was dispersed in a Y-shaped fashion over 30 stations 
Table 1. Overview of prominent SKA straw-man requirements (adapted from [4]) as compared to a typical (hypothetical) Radio Telescope array of to day.

\section{Typical RT-array today}

Paraboloidal reflectors (prime and sec. focus) arrays say, $20 \times 25 \mathrm{~m}$ diam.

Collecting Area $<10.000 \mathrm{~m}^{2}$

Multi (narrow) band freq. Bands, dual pol. $0.075-20 \mathrm{GHz}$

$T_{\text {sys }}<150-25 \mathrm{~K}(0.2-20 \mathrm{GHz})$

Few bit ADC's

Multimode 256.000 channel dig. correlator, $>10^{3}$ spectral channels. Simultaneous freq. bands \& subarraying

Tel. Management System

Astronomical Image Processing System ++

\section{SKA (added) requirements}

Antenna concepts being studied

Collecting Area Collecting Area $10^{6} \mathrm{~m}^{2}$. \# stations $>30$ stations, Surface Br. $1 \mathrm{~K} @ 0.1$ " (Contin.)

Multi-band instrument i.e., from 0.03 $-20 \mathrm{GHz}$ Instant. BW: $0.5+\mathrm{f} / 5$ $\mathrm{GHz}, 2$ widely sep. freq. bands

Increased sensitivity $A_{\text {eff }} / T_{\text {sys }} \sim$ $10^{4}$

Multibit (> 12 bit) ADC's

Multibeam i.e., 100 instant. Beams $10^{4}$ sepctral channels with otherwise at least similar backend performance

Blind, on line quality assessment

Improved RFI robustness

Improved dynamic range $>10^{6} @ 1.4$

$\mathrm{GHz} \&$ mapping capability

Polarization purity (image) $40 \mathrm{~dB}$

Coverage: $2 \pi$ Sterad., FOV: 1 sq. degree @ $1.4 \mathrm{GHz}$, Ang. Resol.: $0.1 " @ 1.4 \mathrm{GHz}$

with an approximate diameter of $300 \mathrm{~m}$ each, with a main core in a $3050 \mathrm{~km}$ radius and a small number of outstations up to $150 \mathrm{~km}$. The number of stations will likely increase [6] as the geometric arrangement and location are dictated by imaging, spatial resolution and frequency arguments among others all being elements of study. The physical realisation of each station for a desirable effective receiving area can in principle be near infinite. For reasons including 
the technologies of today instruments, the major concepts so far are limited to about six:

- a large spherical reflector pursued by BAO in China [7]. Efforts concentrate on a first, single station called FAST of $500 \mathrm{~m}$ diameter with a unique adaptive surface. The beam (like Arecibo $300 \mathrm{~m}$ dish in Puerto Rico) can be steered within limits by moving the receiving system over a limited focal range. For FAST possibly coming into operation within ten years, the efficiency and steerability is improved because the adaptive surface preserves as much as possible a parabolic shape.

- low cost $2025 \mathrm{~m}$ paraboloids promoted by the Indian NCRA. In order to reduce weight and cost and along the lines of the new Giant Meter Radio Telescope (GMRT) $45 \mathrm{~m}$ dishes, the concept uses tensioned wires to preserve the proper shape of the main structural surface elements.

- many mass produced small diameter (about $5 \mathrm{~m}$ ) antennas by the US SETI institute. As a result of recent studies for near future SETI, efforts are now concentrated to the realisation of the 1 hectare telescope (now called Allen Telescope Array or TA coming into operation within 4-5 years [8].

- a new concept of a large adaptive reflector (AR antenna in which the receiver(s) are located in a teetered balloon above an adaptive groundbased reflector with large F/D (allowing a focal plane array) by the Canadian DRAO [9].

- the electrically steerable active array antennas by the Dutch ASTRON and combinations of these e.g. hybrid arrays. Wideband, wavelength sampled active electronic receive arrays are new to radio astronomy emphasising the need for intense R\&D. Contrasting the mechanical arrays, this approach relies on electronic cost equations and provides huge flexibility at the expense of a larger complexity. So far, this has resulted in several demonstrators including work toward a thousand element multibeam (32 beams) array THEA to be operational for testing [10] end 2000.

- Other solutions e.g. focal plane arrays by ATNF in Australia, are part of active research and more recently, the study of a Luneberg Lens array concept [11].

- More advanced possibilities may be different e.g. a 3D frequency selective surface with electronically or optically controlled reconfigurable dipole elements [12].

Based on astronomical requirements, SKA is now seen as three-decade instrument (see Table 1) and probably not based on a single antenna concept and integration of technologies. A combination of different technologies of say, a decade bandwidth each, could optimise SKA e.g. with respect to astronomy, technology, and costs while beneficial in the international framework. SKA would then constitute the total of the low-, mid-, and high-band instruments.

A recent study was done on a dedicated wide band low frequency array (LOFAR) ranging from 15 to over $150 \mathrm{MHz}$ of electrically short active dipole 


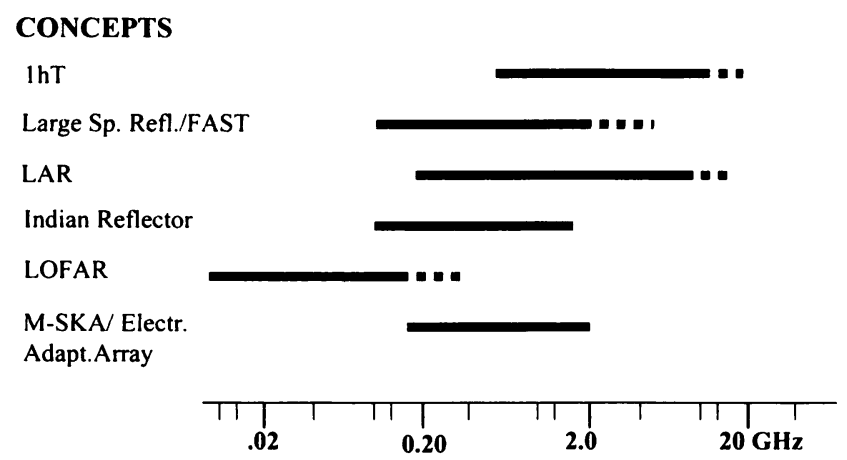

Figure 1. Presently studied concepts versus expected optimum frequency range $(\mathrm{GHz})$ for the different concepts

antennas configured as a sparse electronically steered array [13]. Signal processing e.g. multibeaming, calibration and interference mitigation techniques is overlapping with aspects for the mid- range (say from $0.2-2 \mathrm{GHz}$ ) electronic array SKA R \& D program at ASTRON[10]. Because LOFAR is expected to be operational in 5-6 years time, it will provide an excellent early testbed.

For the mid-range electronic array concept, the maximum frequency is about $2 \mathrm{GHz}$ as the number of active antenna elements of this coherently sampled aperture antenna becomes prohibitively large otherwise. Combined with the argument of skynoise and achievable receiver noise, it is probably realistic to say that below about $1.5 \mathrm{GHz}$ a good case can be made for aperture (phased) array antennas.

The ndianparaboloidal reflector antenna, when made cheap as a further development of the GMRT dishes, will be primarily suitable for the low- and mid-band SKA say from about $50 \mathrm{MHz} 1.5 \mathrm{GHz}$ due to the large gaps in the wire mesh.

On the opposite part of the spectrum, the ATA dishes due to their small size of about $45 \mathrm{~m}$ probably inhibits observations below about $0.5 \mathrm{GHz}$. Their solid surface makes the ATA concept best suitable to cover the mid- and high band SKA. The large prime focus spherical reflector dish concept can operate from $100 \mathrm{MHz}$ but will not be suitable much beyond about $2 \mathrm{GHz}$. The prime focus LAR concept aim to operate from a few hundred up to about $10 \mathrm{GHz}$ and possibly higher frequencies possibly using aperture feed arrays. These mainstream concepts today vs. their expected nominal frequency range are summarised in Figure 1. Together with considerations like expected performance, technology, cost and maximum science return, maintainability and location/site among others, a mechanism to conclude on the final (choice of) concept(s) must be set 
up around 2005. Before that time, these and other as of today newer concepts, must engage in active $R \& D$ programs addressing at least some if not all of the major challenges.

\section{Some Technology Challenges}

SKA will operate as a wideband low noise polarimetric interferometer. Paraboloidal or spherical reflector receiving systems come closest to extrapolating performance of today wide band systems and given appropriate modelling tools at least intuitively, seems doable from the technology maturity point of view. The implementation of the 100 or so required simultaneous beams is however nontrivial. With the appropriate feed design, these systems have the advantage that the collecting area can be constant over frequency. More than one feed may be required for optimal performance but most challenges are embedded in a single advanced receiver per telescope within a raditionalmechanical platform.

This is contrasting the concept of electronically scanned aperture arrays. Relevant also for the military and telecom e.g. base-station applications in general, the emphasis remains largely on technology and the issues are about identifying enabling technologies, their trends and the integration of technologies and functions. Furthermore, the effective area associated with a single basic element antenna in this concept is decreasing with increasing frequency. In order to maintain a large effective area over a decade bandwidth, the aperture must be synthesised with the minimum number of elements say 2 or 3 each for a different frequency range within the total decade. $\mathrm{R} \& \mathrm{D}$ is toward finding a suitable element arrangement for dual polarisation with a power law vs. frequency close to 0 [14]. Subsequent studies model the multi-element arrangement just mentioned [10] showing some preliminary work) and to find a low cost architecture that includes the active low noise receiving part.

Assuming that the project investment as a whole should not exceed today 600 MUS $\$$ limit, the cost per square meter is required to be of order a few hundred US $\$$ or less for all concepts studied. This is over an order of magnitude lower than paraboloidal reflectors commonly in use now and a few times less than the GMRT. Because of the many receiving elements for the electronic adaptive array, the frontend (package) is likely the area where cost, power, signal distribution, reliability and packaging are most important considerations and cooling to achieve lowest noise figures is not likely. Conventional (e.g. Pulse Tube with no moving parts) cooling systems are useful in large reflector systems while emerging low cost cryogenic technologies for mass market applications now considered for high speed computers, may widen the application to other SKA concepts. In any case, over time, the approach should reflect the rapid pace of technology change and consider the modularity vs. upgradability issue.

\section{Conclusion}

Different concepts for the Square Kilometer Array are actively studied in various countries involving new approaches not used before in radio astronomy and holding promise to make a significant contribution to SKA. A framework involving different countries has been defined that allows optimum use of the R\& D work, 
a better understanding of the astronomical requirements and in time, leading to a concept choice. So far, after 5 years of study, no major stumbling blocks have been identified but at the same time further R \& $D$ is required. Partly as a spin off before SKA will become a reality, a large diameter single dish telescope (FAST) and other arrays (LOFAR and ATA) may become operational. As an educational spin-off, a new generation of radio technologists and astronomers alike will find a vehicle for many years of challenging $R \& D$ up to the first real observations.

\section{References}

Bregman, J., This Conference, IAU 199, Dec. 1999 and Proc. SPIE, 19-32, Munich, March 2000

Carlson, B., et al.; Large Adaptive Reflector; a $200 \mathrm{~m}$ diameter Radio Telescope, Proc. SPIE 4015, 33-44, Munich, March 2000

Dreher, J.W., he One Hectare Telescope (1hT) Project, Ibid.

James, G., et. al. ; uneberg lens element for the SKA, Proc. SPIE 4015, 410419, Munich, March 2000

Lonsdale, C., Concepts for a Large-N SKA Ibid.

Noordam J., alibrating SKA: a Challenge!, Persp. on Radio Astronomy: Techn. for Large Ant. Arrays, Eds. A.B. Smolders, M. van Haarlem, ISBN 90-805434-2-X, April 1999

Peeters Weem, J., Notaros, B.M., Popovic, Z., Roadband Element Array Considerations for SKAand ref., Persp. on Radio Astronomy: Techn. for Large Ant. Arrays, Eds. A. B. Smolders, M. van Haarlem, ISBN 90805434-2-X, April 1999

Peng B., et.al.; The Technical scheme for FAST Ibid.

Perspectives on Radio Astronomy: Science with Large Antenna Arrays, Ed. M. van Haarlem,, ISBN 90-805434-1-1, April,1999

Schaubert, D.H. Chio, T.H., Wideband Vivaldi Arrays for Large Aperture Antennas, Persp. on Radio Astronomy: Techn. for Large Ant. Arrays, Eds. A.B.Smolders, M. van Haarlem, ISBN 90-805434-2-X, April 1999

Science with the Square Kilometre Array, Ed. A. R.Taylor and R. Braun, Calgary, 1999 and the Workshop on Large Antennas, Univ. of Alberta, Calgary, Aug. 1998

URSI Large Telesc. Work.Gr.\& 1kT Intern. Techn. Workshop, ATNF-CSIRO, Sydney, Australia, Dec 1997

van Ardenne, A., et. al.; Active Adaptive antenna for Radioastronomy; results for the SKA R \& D program, Proc. SPIE 4015, 420- 433, Munich, March 2000

van Ardenne, A., The Technology Challenge for the Next Generation Radiotelescopes Persp. on Radio Astronomy: Technologies for Large Antenna Arrays, Eds. A.B. Smolders, M. van Haarlem, ISBN 90-805434-2-X, April 1999 